JOURNAL OF THE

AMERICAN MATHEMATICAL SOCIETY

Volume 13, Number 1, Pages 125-127

S 0894-0347(99)00319-7

Article electronically published on September 29, 1999

\title{
UN LEMME DE DÉPLACEMENT POUR LES ZÉRO-CYCLES SUR LES FIBRATIONS EN CONIQUES (APPENDICE À L'ARTICLE DE J.-L. COLLIOT-THÉLÈNE)
}

\author{
E. FROSSARD ET V. SURESH
}

Dans l'article précédent, J.-L. Colliot-Thélène utilise un lemme de déplacement et d'effectivité de zéro-cycles sur les surfaces fibrées en coniques au-dessus d'une courbe de genre quelconque (Prop. 3.3 de l'article). Dans cet appendice, nous donnons la démonstration de ce résultat. La méthode que nous employons ici, dans le cas où le corps de base est de caractéristique nulle, utilise certaines courbes auxiliaires sur les surfaces considérées, courbes sur lesquelles on applique le théorème de RiemannRoch. L'existence de ces courbes auxiliaires est donnée par le théorème suivant, dû à Coray ([Co80], voir aussi Kleiman-Altman [Kl/Al79]) .

Théorème (Coray). Soit $k$ un corps parfait infini. Soit $X \subset \mathbf{P}_{k}^{n}$ une k-variété lisse, projective, géométriquement intègre de dimension supérieure ou égale à 1 . Soit $\left\{P_{1}, \cdots, P_{r}\right\}$ un ensemble fini de points fermés de $X$. Alors il existe une $k$ courbe $V \subset X$, lisse, projective, géométriquement intègre, qui passe par les points $P_{1}, \cdots, P_{r}$. De plus on peut construire $V$ comme étant l'intersection de $X$ avec une hypersurface de $\mathbf{P}_{k}^{n}$ de degré $d+1$, où $d=\sum_{i=1}^{i=r} \operatorname{deg}_{k}\left(k\left(P_{i}\right)\right)$.

Rappelons par ailleurs un résultat démontré par Salberger dans sa thèse ([Sal85], Part [b], Theorem 3.5).

Théorème (Salberger). Soit $k$ un corps parfait. Soit $\pi: X \rightarrow C$ une surface fibrée en coniques au-dessus d'une $k$-courbe $C$. Appelons $g$ le genre de la courbe $C$. Notons $r$ le nombre de fibres géométriques singulières de la fibration $\pi: X \rightarrow C$. Alors tout zéro-cycle sur $X$ de degré supérieur ou égal à $d_{0}=2 g+\sup (0,(r-3) / 2)$ est rationnellement équivalent à un zéro-cycle effectif sur $X$.

Ce résultat est le point de départ de la démonstration de la proposition qui nous intéresse ici.

Proposition. Soit $k$ un corps de caractéristique 0 . Soit $\pi: X \rightarrow C$ une surface fibrée en coniques au-dessus d'une $k$-courbe $C$ et soit $\left\{P_{1}, \cdots, P_{n}\right\}$ un ensemble fini de points fermés de $C$. Il existe un entier $N$ tel que pour tout corps $L$ contenant $k$ on ait la propriété suivante: tout zéro-cycle sur $X \times_{k} L$ de degré au moins égal à $N$ est rationnellement équivalent à un zéro-cycle effectif $z$ sur $X \times_{k} L$ tel que $\left(\pi_{L}\right)_{*}(z)$ ait son support étranger à $P_{1}, \cdots, P_{n}$ et ait toutes ses multiplicités égales à 1 .

Démonstration. Pour simplifier les notations appelons $Z \subset X$ la réunion des fibres $X_{i}=\pi^{-1}\left(P_{i}\right), 1 \leq i \leq n$. Nous allons procéder en deux étapes.

Received by the editors May 29, 1998 and, in revised form, June 17, 1999.

1991 Mathematics Subject Classification. Primary 14C15; Secondary 14C25.

(C)1999 American Mathematical Society 
Etape 1. Fixons d'abord quelques données, indépendantes du corps de base. Soit $g$ le genre de la courbe $C$. Soit $r$ le nombre de fibres géométriques singulières de la fibration $\pi: X \rightarrow C$. Notons $d_{0}=2 g+\sup (0,(r-3) / 2)$. Soit $\chi$ la caractéristique d'Euler-Poincaré du faisceau $\mathcal{O}_{X}$. Soit $s=\sum_{i \in I} \operatorname{deg}_{k}\left(k\left(P_{i}\right)\right)+2 \sum_{i \in J} \operatorname{deg}_{k}\left(k\left(P_{i}\right)\right)$ où $I \subset\{1, \cdots, n\}$ décrit les indices $i$ tels que $X_{i}$ est singulière et où $J$ est le complémentaire de $I$. Soit $j: X \hookrightarrow \mathbf{P}_{k}^{m}$ un plongement et soit $\chi^{\prime}$ la caractéristique d'Euler-Poincaré du faisceau $j^{*}\left(\mathcal{O}_{\mathbf{P}_{k}^{m}}(-s-1)\right)$. Posons $h=1-\chi+\chi^{\prime}$. Nous allons déterminer en fonction de ces données un entier d tel que tout zéro-cycle sur $X$ de degré supérieur ou égal à d est rationnellement équivalent à un zéro-cycle effectif sur $X$ ayant son support en dehors de $Z$.

Pour tout $i \in\{1, \cdots, n\}$, choisissons $M_{i}$ un point de degré minimal dans la fibre $X_{i}$. Soit $V \subset X$ une $k$-courbe lisse, projective, géométriquement intègre, qui passe par les points $M_{1}, \cdots, M_{n}$, telle que $V$ est l'intersection de $X$ avec une hypersurface de $\mathbf{P}_{k}^{m}$ de degré $s+1$. Une telle courbe existe d'après le théorème de Coray cité ci-dessus. La courbe $V$ est alors de genre $h$. Posons $d=d_{0}+2 h \operatorname{deg}_{k}\left(k\left(M_{1}\right)\right)$. Soit $z$ un zéro-cycle sur $X$ de degré supérieur ou égal à $d$. Soit $z^{\prime}=z-2 h M_{1}$. Le cycle $z^{\prime}$ étant de degré supérieur ou égal à $d_{0}$, il est rationnellement équivalent sur $X$ à un cycle effectif. Les fibres $X_{i}$ étant des coniques, les groupes $A_{0}\left(X_{i}\right)$ sont nuls (rappelons que $A_{0}\left(X_{i}\right)$ désigne le groupe des zéro-cycles de degré 0 de la variété $X_{i}$, modulo l'équivalence rationnelle). De plus comme chaque point $M_{i}$ est un point de degré minimal dans la fibre $X_{i}$, tout zéro-cycle effectif sur $X$ est la somme de multiples positifs des $M_{i}$ et d'un zéro-cycle effectif à support en dehors de $Z$. Ainsi $z$ est rationnellement équivalent à $z_{1}+z_{2}$, où $z_{1}$ est un zéro-cycle effectif à support en dehors de $Z$, et où $z_{2}=2 h M_{1}+\sum_{i=1}^{i=n} a_{i} M_{i}$ avec $a_{i} \geq 0$ pour tout $i$. D'après le théorème de Riemann-Roch appliqué sur la courbe $V$, le cycle $z_{2}$ ayant un degré supérieur ou égal à deux fois le genre de $V$, le système linéaire associé est sans point de base (voir [Ha77], IV.3.2). Le cycle $z_{2}$ est donc lui-même rationnellement équivalent sur $V$ à un cycle effectif dont le support est en dehors de $V \cap Z$. Ceci prouve bien que l'entier $d$ a la propriété requise.

Etape 2. Nous allons maintenant déterminer un entier $N$ tel que tout zéro-cycle sur $X$ de degré supérieur ou égal à $N$ est rationnellement équivalent à un zéro-cycle effectif sur $X$ ayant son support en dehors de $Z$ et dont l'image par $\pi_{*}$ a toutes ses multiplicités égales à 1. Montrons d'abord qu'il existe un point $e$ de degré 2 sur $k(C)$ dans la fibre générique de $\pi$, de corps résiduel $\kappa / k(C)$, de telle sorte que $k$ est algébriquement clos dans $\kappa$. Soit $K=k(C)$ et soit $a, b \in K^{*}$ tels que la fibre générique de $\pi$ est la conique d'équation $X^{2}-a Y^{2}-b T^{2}=0$. Supposons que $a$ (ou $b$ ) n'est pas le produit d'un élément de $k^{*}$ par un carré de $K^{*}$. Alors $k$ est algébriquement clos dans l'extension $K(\sqrt{-a}) / K$ (ou $K(\sqrt{-b}) / K$ ) et nous pouvons prendre $e$ correspondant à cette extension. Sinon nous pouvons supposer que $a$ et $b$ sont tous deux éléments de $k^{*}$. Fixons $\lambda \in K^{*}$ un élément transcendant sur $k$. Puisque l'extension $\left(\bar{k} \otimes_{k} K\right) / \bar{k}(\lambda)$ est finie, il n'existe qu'un nombre fini d'éléments de $\bar{k}(\lambda)^{*} /\left(\bar{k}(\lambda)^{*}\right)^{2}$ qui deviennent triviaux dans $\left(\bar{k} \otimes_{k} K\right)^{*} /\left(\left(\bar{k} \otimes_{k} K\right)^{*}\right)^{2}$. Par ailleurs il est clair que si $c$ et $c^{\prime}$ sont deux éléments distincts de $k^{*}$, les éléments $a(\lambda+c)^{2}+b$ et $a\left(\lambda+c^{\prime}\right)^{2}+b$ n'ont pas la même classe dans $\bar{k}(\lambda)^{*} /\left(\bar{k}(\lambda)^{*}\right)^{2}$ (il suffit de regarder les zéros de ces deux fonctions). Ainsi, puisque $k$ est infini, il existe $c \in k^{*}$ tel que $f=a(\lambda+c)^{2}+b$ n'est pas un carré dans $\bar{k} \otimes_{k} K$. Maintenant $k$ est algébriquement clos dans l'extension $K(\sqrt{-f}) / K$ et il existe un point $e$ de degré 2 dans la fibre générique, de corps résiduel $\kappa=K(\sqrt{-f})$. 
Soit $E$ l'adhérence schématique de $e$ dans $X$. C'est une $k$-courbe projective, géométriquement intègre et $\pi$ induit un morphisme $p: E \rightarrow C$ qui est fini et plat de degré 2 . Sans nuire à la généralité, nous pouvons supposer que l'ensemble $\left\{P_{1}, \cdots, P_{n}\right\}$ contient tous les points de $C$ images par $p$ de points singuliers de $E$. Soit $g^{\prime}$ le genre de la normalisée de $E$. Fixons $Q$ un point régulier de $E$ et notons $q$ son degré sur $k$. Soit $d$ un entier comme dans l'étape 1. Posons $N=d+\left(2 g^{\prime}+1\right) q$. Soit $z$ un zéro-cycle sur $X$ avec $\operatorname{deg}(z) \geq N$. D'après ce qui a été montré dans l'étape 1 , le cycle $z-\left(2 g^{\prime}+1\right) Q$ est rationnellement équivalent à un zéro-cycle $z^{\prime}$ effectif sur $X$ à support en dehors de $Z$. Soit $P$ un point fermé de $C$. Puisque $A_{0}\left(X_{P}\right)=0$ et que $E$ est de degré 2 sur $C$, soit il y a un point $M$ dans $X_{P} \cap E$ tel que $[k(M): k(P)]=1$ et le zéro-cycle $\left.z^{\prime}\right|_{X_{P}}$ est rationnellement équivalent à un multiple positif de $M$, soit il y a un point $M$ dans $X_{P} \cap E$ tel que $[k(M): k(P)]=2$ et le zéro-cycle $\left.z^{\prime}\right|_{X_{P}}$ est rationnellement équivalent à la somme d'un multiple positif de $M$ et d'au plus un point de $X_{P}$ ayant le même degré que $P$. Nous voyons ainsi que $z^{\prime}$ est rationnellement équivalent à $z_{1}+z_{2}$ où $z_{1}$ et $z_{2}$ sont des zéro-cycles effectifs à support en dehors de $Z$, le cycle $\pi_{*}\left(z_{1}\right)$ a toutes ses multiplicités égales à 1 et le cycle $z_{2}$ est supporté par $E$. Nous appliquons maintenant le lemme 3.1 de [CT99] au morphisme $p: E \rightarrow C$ et au cycle $z_{2}+\left(2 g^{\prime}+1\right) Q$ qui a son support dans le lieu régulier de $E$ par notre hypothèse. Notons que si $E$ n'est pas lisse, ce lemme est encore valable pour un cycle à support dans le lieu régulier de $E$ (on peut passer à la normalisée de $E$ ). Puisque $\operatorname{deg}\left(z_{2}+\left(2 g^{\prime}+1\right) Q\right) \geq 2 g^{\prime}+1$, on déduit de ce lemme que $\left(z_{2}+\left(2 g^{\prime}+1\right) Q\right)$ est rationnellement équivalent sur $E$ à un cycle $z_{3}$ avec $\pi_{*}\left(z_{3}\right)$ ayant toutes ses multiplicités égales à 1 et étant à support en dehors de $P_{1}, \cdots, P_{n}$ et de $\pi_{*}\left(z_{1}\right)$. Alors le zéro-cycle $z_{1}+z_{3}$ possède la propriété demandée et est rationnellement équivalent à $z$.

Par ailleurs il est clair que si l'on fixe une $k$-courbe $E$ comme ci-dessus et un ensemble de points fermés $P_{1}, \cdots, P_{n}$ de $C$ (contenant tous les points de $C$ images par $p$ de points singuliers de $E$ ), l'entier $N$ calculé ci-dessus satisfera encore la propriété demandée après extension du corps de base $k$, ce qui achève la démonstration de la proposition.

\section{REFERENCES}

[CT99] J.-L. Colliot-ThÉLÈne, Principe local-global pour les zéro-cycles sur les surfaces réglées, J. Amer. Math. Soc., ce volume.

[Co80] D. F. Coray, Two remarks on the Bertini theorem, tapuscrit (1980).

[Ha77] R. Hartshorne, Algebraic Geometry, Graduate Texts in Mathematics, Springer (1977). MR 57:3116

[Kl/Al79] S. L. Kleiman and A. B. Altman, Bertini theorems for hypersurface sections containing a subscheme, Communications in Algebra 7 (8), 775-790 (1979). MR 81i:14007

[Sal85] P. Salberger, K-theory of orders and their Brauer-Severi schemes, Thèse, Université de Göteborg, 1985.

Université de Caen, Campus II, Département de Mathématiques, Laboratoire SDAD, ESA 6081, Boulevard du Maréchal Juin, 34095, B.P. 5186, F-14032 Caen Cédex, France

E-mail address: efrossard@math.unicaen.fr

School of Mathematics, Tata Institute of Fundamental Research, Homi Bhabha ROAD, BOMBAy 400 005, India

E-mail address: suresh@math.tifr.res.in 